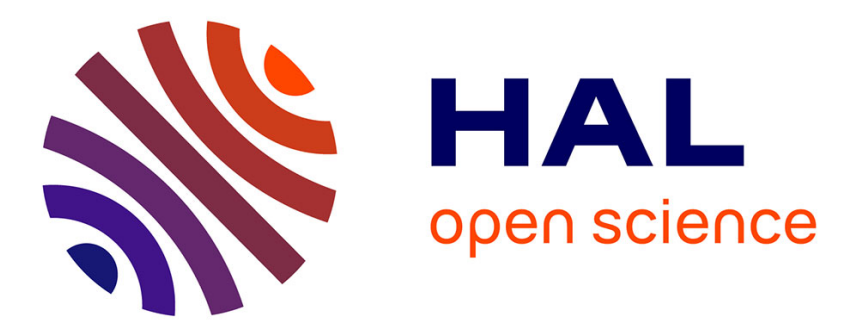

\title{
Les lignées d'addition blé-Aegilops ventricosa. V. - Caractérisation des lignées et de leurs progéniteurs au moyen des électrophorégrammes des $\beta$ amylases et des peroxydases
}

Françoise Dosba, Philippe Joudrier, Karoly Kobrehel, Anne-Marie Tanguy, Marguerite Michel, Ginette Gobin, Anne Guirao

\section{To cite this version:}

Françoise Dosba, Philippe Joudrier, Karoly Kobrehel, Anne-Marie Tanguy, Marguerite Michel, et al.. Les lignées d'addition blé-Aegilops ventricosa. V. - Caractérisation des lignées et de leurs progéniteurs au moyen des électrophorégrammes des $\beta$ amylases et des peroxydases. Agronomie, 1983, 3 (6), pp.569-576. hal-00884545

\section{HAL Id: hal-00884545 https://hal.science/hal-00884545}

Submitted on 1 Jan 1983

HAL is a multi-disciplinary open access archive for the deposit and dissemination of scientific research documents, whether they are published or not. The documents may come from teaching and research institutions in France or abroad, or from public or private research centers.
L'archive ouverte pluridisciplinaire HAL, est destinée au dépôt et à la diffusion de documents scientifiques de niveau recherche, publiés ou non, émanant des établissements d'enseignement et de recherche français ou étrangers, des laboratoires publics ou privés. 


\title{
Les lignées d'addition blé-Aegilops ventricosa.
} V. - Caractérisation des lignées et de leurs progéniteurs au moyen des électrophorégrammes des $\beta$ amylases et des peroxydases

\author{
Françoise DOSBA $\left({ }^{1}\right)$ Philippe JOUDRIER * \& Karoly KOBREHEL * \\ avec la collaboration technique d'Anne-Marie TANGUY, Marguerite MICHEL *, Ginette GobIN* \& Anne \\ GUIRAO * \\ I.N.R.A., Station d'Amélioration des Plantes, Centre de Recherches de Rennes, B.P. 29 F35650 Le Rheu. \\ * I.N.R.A. Laboratoire de Technologie des Céréales, 9 place Viala, F 34060 Montpellier Cedex.
}

\begin{abstract}
Afin de caractériser les chromosomes du génome $M^{\prime}$ d'Aegilops ventricosa, des marqueurs biochimiques cnzymatiques ( $\beta$ amylases et peroxydases) ont été étudiés chez différentes espèces d'Aegilops (fig. 3 ) et chez des lignées d'addition blé - Aegilops ventricosa.

En ce qui concerne les $\beta$ amylases, la plupart des lignées d'addition analysées possèdent un zymogramme correspondant à celui du blé tendre utilisé. Cependant des types de zymogramme particuliers (types VIII à X) sont observés chez quelques lignées (tabl. 1 et 2, fig. 1).

Dans le cas des peroxydases, la majorité des lignées d'addition ont également le zymogramme caractéristique du blé tendre utilisé. Par ailleurs, les lignées ayant plutôt un diagramme typique d'Aegilops ventricosa ou de l'amphiploïde ne possèdent pas de chromosomes additionnels (tabl. 3, fig. 4).

Une analyse plus approfondie des différents types enzymatiques observés (pour les deux enzymes) à partir de matériel bien défini chromosomiquement révèle une hétérogénéité structurale de ces lignées plutôt qu'un marquage chromosomique (fig. 2).
\end{abstract}

Mots clés additionnels : Blé tendre, Triticum aestivum, Aegilops, Hybridations interspécifiques, Marquage chromosomique.

Additional key words : Soft wheat, Triticum aestivum, Aegilops, Interspecific hybridization, Chromosomal markers.

\section{INTRODUCTION}

Dans le domaine végétal, on assiste depuis une dizaine d'années à une mise en évidence croissante de polymorphismes biochimiques, tant protéiques qu'enzymatiques.

( $)$ Adresse actuelle: I.N.R.A., Station de Recherches d'Arboriculture fruitière, Centre de Recherches de Bordeaux, F 33140 Pont-de-la-Maye.
Outre les études visant à une meilleure connaissance de leur variabilité et de leur contrôle génétique, se sont développées des recherches les utilisant en tant que marqueurs. L'utilisation de marqueurs biochimiques se révèle particulièrement intéressante chez les Triticinées car les sélectionneurs ont de plus en plus recours à l'hybridation interspécifique pour introduire des caractéristiques agronomiques diverses. Ces marqueurs permettent en effet, 
d'identifier les chromosomes et, ce faisant, apportent des renseignements sur les homéologies chromosomiques et, en général, sur l'éloignement génétique des espèces (BRODY \& MENDLINGER, 1980). De telles études ont déjà été réalisées sur les hybrides blé $\times$ seigle (BERNARD et al., 1977 ; IRANI \& BHATIA, 1972), blé $\times$ Agropyron (CAUDERON et al., 1978 ; NAGAYOSHI \& MOCHIZL KI, 1971 ; KOBREHEL, 1978 ; KONAREV, 1979), blé $\times$ orge (ISLAM et al., 1978 ; HART et al., 1980) et également blé $\times$ Aegilops (BROWN et al., 1979 ; CALDWELl \& KASARDA, 1978).

En ce qui concerne les lignées d'addition blé $\times$ Aegilops ventricosa, une étude récente de DELIBES et al. (1981) a mis en évidence l'existence de 2 marqueurs - l'un protéique, l'autre enzymatique correspondant à la synthèse d'une phosphatase alcaline - qui sont liés à la présence de 2 chromosomes additionnels de l'Aegilops. Par ailleurs, l'analyse des électrophorégrammes des gliadines de ces mêmes lignées n'a permis de présumer que du marquage d'une seule lignée (DOSBA \& AUTRAN, 1983).

Dans le même esprit, nous avons utilisé d'autres systèmes enzymatiques ( $\beta$ amylases, peroxydases) en vue de caractériser Ae. veniricosa, ses progéniteurs et les lignées d'addition blé $\times$ Ae. ventricosa.

\section{MATÉRIEL ET MÉTHODES}

\section{A. Matériel d'étude}

\section{Analyse des $\beta$ amylases}

Les matériels étudiés sont constitués de :

- 4 souches d'Aegilops uniaristata Vis. $(2 \mathrm{n}=14$, génome $\mathbf{M}^{\mathrm{u}}$ ) numérotées de 1 à 4 .

- 2 souches d'Ae. comosa Sibth et Sm. $(2 \mathrm{n}=14$, génome $\mathrm{M}) \mathrm{n}^{\mathrm{os}} 1$ et 3 .

- 3 souches d'Ae. squarrosa L. $(2 \mathrm{n}=14$, génome $\mathrm{M})$ $\mathrm{n}^{\text {os }} 38,39,40$.

- 11 souches d'Ae. ventricosa Tausch. ( $2 \mathrm{n}=28$, génomes $\left.D^{v} M^{v}\right) n^{\circ} 1,2,3,4,5,8,10,11,19,41$ et 188 .

- les géniteurs des lignées d'addition : la souche d'Ae. ventricosa Tausch $\mathrm{n}^{\circ} 11$; un cultivar de blé dur, Triticum aethiopicum Jakubz $\mathrm{n}^{\circ} 1 \mathrm{~A}(2 \mathrm{n}=28$, génomes $\mathrm{AB})$; la variété «Moisson» de blé tendre, T. aestivum (L.) Thell., ssp. vulgare (Vill.) M.K. ( $\mathrm{n}=42$, génomes $\mathrm{ABD})$.

- l'amphiploïde $\mathrm{n}^{\circ}$ A $2(2 \mathrm{n}=56$; génomes $\mathrm{ABD}^{\prime} \mathrm{M}^{\prime}$ ), issu du croisement (Ae. ventricosa $\mathrm{n}^{\circ} 11 \times T$. aethiopicum $\mathrm{n}^{\circ} 1 \mathrm{~A}$ ).

- 69 lignées d'addition sur cytoplasme «ventricosa» (lignée v) issues du croisement (Ae. ventricosa $\mathrm{n}^{\circ} 11 \times T$. aethiopicum 1 A) $\times T$. aestivum $\mathrm{cv}$. "Moisson" " suivi de 4 à 8 générations d'autofécondation selon l'année d'analyse. Au total 159 descendances de plantes ont été analysées de 1975 à 1980. Quinze lignées ont été analysées à partir de 2 récoltes différentes, 6 à partir de 3 et 1 à partir de 4 récoltes.

- 69 lignées d'addition sur cytoplasme «Moisson» (lignées $\mathrm{m}$ ) résultant de l'hybridation (T. aestivum $\mathrm{cv}$. "Moisson ${ }^{4}$ " $\times$ Ae. ventricosa $\left.\mathrm{n}^{\circ} 11\right) \times T$. aestivum cv. "Moisson ${ }^{4}$ », menées ensuite en autofécondation pendant 1 à 5 ans. 134 descendances de plantes ont été analysées à partir de 5 récoltes (1975-1979). 9 lignées ont été analysées à partir de 2 récoltes différentes, 3 à partir de 3 récoltes.

\section{Analyses des peroxydases}

Le matériel utilisé comprend:

- 4 souches d'Ae. uniaristata $\mathrm{n}^{\mathrm{os}} 1$ à 4.

- 2 souches d'Ae. comosa $\mathrm{n}^{\mathrm{os}} 1$ et 3 .

- 9 souches d'Ae. squarrosa $\mathrm{n}^{\circ \mathrm{s}} 9,12,15,20,32,33,38$, 39 , 40. Les souches 12 et 20 sont tétraploïdes $(2 \mathrm{n}=28$, génomes D D).

- 3 souches d'Ae. ventricosa $n^{\text {os }} 7,10,11$.

- Les géniteurs des lignées d'addition et l'amphiploïde $\mathrm{A}_{2}$ décrits précédemment.

- 69 lignées d'addition $v$ réparties dans 142 descendances de plantes analysées de 1976 à 1979 . Treize lignées ont ćté étudiées à partir de 2 récoltes différentes, 5 à partir de 3 récoltes.

- 62 lignées $m$ représentées au cours de cette même période par 92 descendances de plantes. 18 lignées ont été analysées à partir de 2 récoltes et 1 à partir de 3 récoltes.

Les différents numéros des lignées $\mathrm{v}$ et des lignées $\mathrm{m}$ correspondent à plusieurs chromosomes ajoutés et constituent des groupes établis en fonction de caractéristiques morphologiques, agronomiques ou cytologiques (DosBA et al., 1978 ; DosBA, 1982).

\section{B. Méthodes utilisées}

\section{Méthodes cytologiques}

Les grains utilisés pour l'extraction des $\beta$ amylases et des peroxydases sont généralement issus de plantes à 44 chromosomes et présentent une méïose régulière. Afin de détecter le lien éventuel entre le chromosome ou le télosome additionnel et un marquage biochimique, des dénombrements chromosomiques ont été effectués à partir de demi-grains germés, selon la méthode antérieurement décrite (DosBA \& DoussinaulT, 1978). L'analyse des $\beta$ amylases ou peroxydases a été réalisée sur le demi-grain correspondant.

\section{Analyse des $\beta$ amylases}

L'extraction des $\beta$ amylases, leur fractionnement électrophorétique en gel de polyacrylamide et leur révélation spécifique ont été effectués dans des conditions précédemment décrites (JOUDRIER \& BERNARD, 1977).

\section{Analyse des peroxydases}

L'extraction des peroxydases, leur séparation électrophorétique en gel de polyacrylamide et leur révélation spécifique ont été celles décrites par KoBREHEL \& FEILLET (1975). On a également utilisé la nomenclature proposée par ces mêmes auteurs.

\section{RÉSULTATS ET DISCUSSION}

\section{A. $\beta$ amylases}

En plus des 7 types de diagrammes de $\beta$ amylases (I à VII) antérieurement décrits (JOUDRIER \& BERNARD, 1977 ; JOUDRIER, 1980), l'examen des Aegilops et des lignées d'addition blé $\times$ Aegilops nous a conduits à définir 3 nouveaux types: VIII, IX et $\mathrm{X}$ (fig. 1 ).

Le type VIII est caractérisé par l'absence de constituant dans la zone $\mathrm{C}_{3}$ du zymogramme et la présence des isozymes : $\mathrm{C}_{02}, \mathrm{C}_{03}, \mathrm{C}_{1}$ et $\mathrm{C}_{2}$; le type IX se définit lui aussi par l'absence des constituants de la zone $C_{3}$ et la présence 

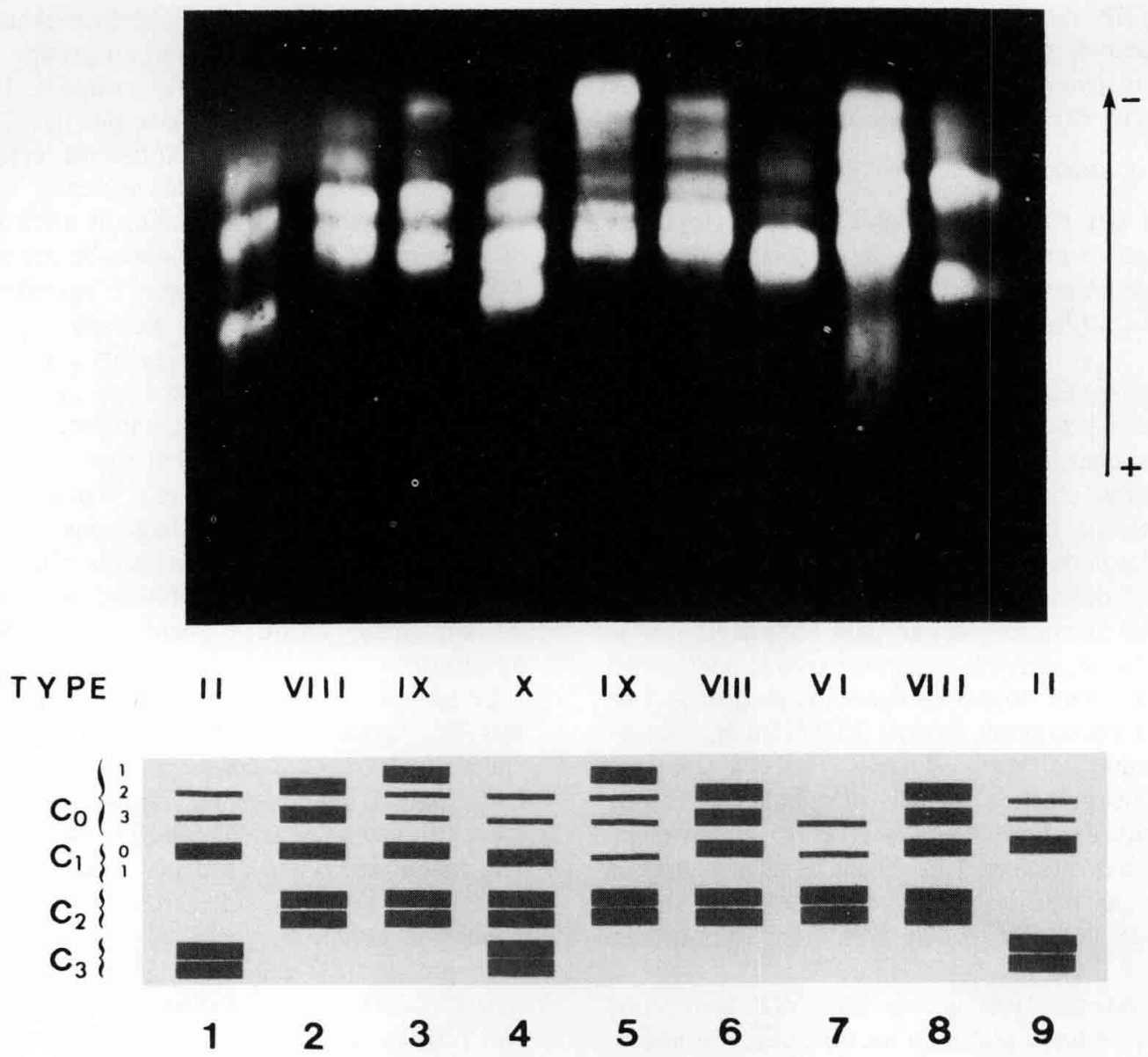

Figure 1

Photographie et schéma correspondant des différents types de zymogrammes $\beta$-amylasiques obtenus chez les lignées d'addition blé - Aegilops ventricosa.

Photograph and corresponding scheme of different types of $\beta$ amylase zymograms from wheat - Aegilops ventricosa addition lines. 1 - "Moisson" (type II)

2 - Lignée d'addition m 12-23 (type VIII)

3 - Lignée d'addition v 246-5 (type IX)

4 - Lignée d'addition v 372-2-10 (tvpe $X$ )

5 - Ae. ventricosa $n^{\prime \prime} 10$ (type $I X$ )

6 - Ae. ventricosa $n^{\prime \prime} 11$ (type VIII)

7 - T. aethiopicum (type $V I)$

8 - Amphiploïde A, (type VIII)

9 - «Moisson» (type II)

des isozymes $\mathrm{C}_{01}$ et $\mathrm{C}_{02}$ (à l'état de trace) $\mathrm{C}_{03}, \mathrm{C}_{1}$ et $\mathrm{C}_{2}$. Le type $\mathrm{X}$ comprend les isozymes suivants: $\mathrm{C}_{02}, \mathrm{C}_{03}, \mathrm{C}_{11}, \mathrm{C}_{2}$ et $\mathrm{C}_{3}$.

\section{Les Aegilops et les blés}

L'analyse des diverses espèces d'Aegilops révèle une hétérogénéité des diagrammes au sein de chaque espèce; c'est le cas des lignées d'Ae. ventricosa. Cependant, 4 souches d'Ae. uniaristata (génome $\mathrm{M}^{u}$ ) ont donné des zymogrammes identiques, caractérisés par l'absence des constituants $\mathrm{C}_{3}$. Bien que le nombre de souches examinées soit faible et que l'origine génétique de ces 4 souches ne soit pas connue, cette absence d'hétérogénéité peut se rapprocher des résultats obtenus au niveau des diagrammes de gliadines par Dosba \& AUTRAN (1983) sur ces mêmes souches et de ceux de KHAKImova (1979) également pour les gliadines mais très vraisemblablement sur d'autres souches. Cette espèce révèle une très faible variabilité sur le plan biochimique.

Les 2 souches d'Ae. comosa (génome M) sont également dépourvues des constituants $\mathrm{C}_{3}$, mais ont des zymogrammes différents.

Les 3 souches d'Ae. squarrosa (génome D) étudiées, de même que la majorité des souches de cette espèce analysées antérieurement (JOUDRIER \& BERNARD, 1977), ont un zymogramme de type VI caractérisé par la présence de constituants $\mathrm{C}_{2}$. Cependant, ces isozymes ne peuvent pas être considérées comme des marqueurs du génome $\mathrm{D}$. On sait en effet par l'étude de leur déterminisme génétique (JOUDRIER, 1980) que la synthèse des constituants $C_{2}$ est contrôlée par le génome $\mathrm{A}$.

En ce qui concerne les Ae. ventricosa (génomes $\mathrm{D}^{\mathrm{v}} \mathrm{M}^{\mathrm{v}}$ ), on n'observe pas d'isozymes dans la zone $C_{3}$ parmi les 11 souches analysées. Des constituants présentant une forte activité dans la zone $\mathrm{C}_{0}$ existent, alors que ces mêmes constituants ont une très faible activité chez les blés. On peut noter que, seule, la souche $\mathrm{n}^{\circ} 11$ est du type VIII, les autres souches se rattachant au type IX (fig. 1).

Le génome $\mathrm{D}$ serait donc plutôt caractérisé par la présence des isozymes du groupe $\mathrm{C}_{3}$ alors que le génome $M$ serait caractérisé par l'ab́sence de constituant dans la zone $\mathrm{C}_{3}$ du zymogramme.

T. aethiopicum $\mathrm{n}^{\circ} 1 \mathrm{~A}$ présente un diagramme caractéristique du type VI antérieurement décrit tandis que "Moisson" se rattache au type II, type le plus fréquemment observé parmi les variétés de blé tendre (AURIAU et al., 1976). L'amphiploïde $\mathrm{A}_{2}$, issu du croisement Ae. ventricosa $11 \times T$. aethiopicum $1 \mathrm{~A}$, présente un zymogramme sem- 
ble à celui des $A e$. ventricosa avec toutefois une différence quantitative d'activité pour les constituants les plus mobiles. Les isozymes majeurs de la zone $\mathrm{C}_{0}$ peuvent donc être considérés comme caractéristiques de cette espèce.

\section{Les lignées d'addition}

a) L'analyse des 69 lignées d'addition sur cytoplasme «Moisson » (lignées $\mathrm{m}$ ) montre que la plupart d'entre elles (59) ont un zymogramme de type II, donc identique à celui de "Moisson"; 10 lignées ont un zymogramme différent (tabl. 1).

Parmi ces dernières, la lignée m 370 présente un diagramme amylasique de type $X$ avec le constituant $C_{11}$ qui est toujours présent chez les plantes à 44 chromosomes de cette lignée. Cependant, elle n'a pas encore été rattachée à un groupe cytogénétique bien défini car elle n'est pas fixée. Au sein des 9 lignées $\mathrm{m}$ du type $\boldsymbol{\beta}$ amylasique VIII, 8 appartiennent au groupe 5 défini par ses caractéristiques cytologiques et agronomiques. Toutefois, on ne peut considérer que le type VIII traduise un marquage du chromosome additionnel de ces lignées. En effet, toutes les lignées $m$ du groupe 5 ne présentent pas un diagramme de type VIII (15 d'entres elles ont un diagramme de type "Moisson ") et des plantes à $2 \mathrm{n}=42$ issues du. groupe 5 (m 294) offrent le diagramme

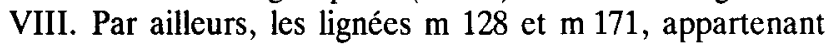
respectivement aux groupes 9 et 7, ont ce même type de zymogramme. De plus les demi-grains à 42,43 ou 44 chromosomes de la lignée m 128 présentent tous le diagramme VIII.

Il est donc vraisemblable que le type VIII révèle une hétérogénéité structurale plutôt qu'un marquage chromosomique.

b) En ce qui concerne les lignées v, le zymogramme II de type «Moisson" est largement observé ; toutefois, un pourcentage élevế de lignées (28 p. 100) présente d'autres types de zymogrammes (tabl. 2).

Les zymogrammes IX et $\mathrm{X}$ sont observés respectivement chez 15 et 5 lignées d'addition $v$. Le diagramme $X$ ne semble pas correspondre à un marquage dû à un chromosome additionnel car on le trouve chez les lignées d'addition (v 86, v 183, v 246, v 263, v 372) appartenant à 3 groupes différents (groupes 4, 5, 7 ; DosBA, 1982), ainsi que chez des plantes à $4 \%$ chromosomes de la lignée v 271 . Le zymogramme IX se rencontre chez 9 lignées du groupe 4 ,
2 lignées du groupe 6 et 3 lignées du groupe 5 et 1 lignée appartenant vraisemblablement au groupe 9.

Parmi les 30 lignées dans le groupe 4, 11 seulement ont un diagramme IX ou $\mathrm{X}$ alors que les 19 autres ont un diagramme II. Or la caractéristique cytologique de cet ensemble de lignées est de présenter un remaniement chromosomique, vraisemblablement entre un chromosome du génome $M^{v}$ et un chromosome du génome $D^{v}$ (DOSBA, 1982). On pourrait penser que le zymogramme IX, assez fréquent dans le groupe 4 et plus spécifique du génome $D^{v}$ de l'Aegilops, traduit chez les lignées la présence d'une translocation réciproque entre un chromosome $\mathrm{D}$ et un chromosome $\mathrm{M}^{\mathrm{v}}$ et que cette translocation différerait quelque peu selon les lignées du groupe 4. Cependant, au sein du groupe 4, les lignées $\vee 27$ et $\vee 30$ ayant même origine ont un diagramme II et IX et le diagramme IX se retrouve aussi dans d'autres groupes de lignées qui n'ont pas ce remaniement chromosomique. En définitive, il est peu probable que les diagrammes IX ou $\mathrm{X}$ soient caractéristiques des lignées du groupe 4.

Le groupe 6, constitué de 3 lignées, présente les diagrammes IX (lignées v 136 et $v$ 137) et II (lignée v 219). Les lignées $v 136$ et $v 137$ posséderaient au moins une partie de l'information génétique du chromosome ajouté dans le groupe 9 (DELIBES et al., 1981). Or, le groupe 9 a un diagramme II. Il est donc peu vraisemblable que le diagramme IX constitue un marquage du chromosome additionnel du groupe 6.

Le groupe 5 , formé de 5 lignées $\mathrm{v}$, a un diagramme de type IX sauf la lignée $v 260$ pourtant bien caractéristique du type. Si ce diagramme était spécifique du chromosome additionnel, on devrait le retrouver dans le groupe 5 des lignées $m$ qui a des diagrammes II ou VIII et qui a le même chromosome additionnel que le groupe 5 des lignées $v$.

Il est vraisemblable que les lignées $v$ ne présentant pas le diagramme de type II manifestent une hétérozygotie structurale dont le génome $\mathrm{D}^{v}$ d'Ae. ventricosa ou ceux de l'espèce pont T. aethiopicum serait à l'origine. En effet, 2 rétrocroisements par «Moisson» seulement ont été réalisés lors de l'extraction des lignées $\mathrm{v}$.

Tant chez les lignées $v$ que chez les lignées $m$, on rencontre des zymogrammes $\boldsymbol{\beta}$ amylasiques voisins de celui d'Ae. ventricosa et jamais encore rencontrés chez les Triticum. Il est actuellement difficile de dire que ces

TABLEAU 1

Caractéristiques des lignées $m$ ayant un diagramme $\beta$-amylasique différent de celui de "Moisson ". Characteristics of $m$ addition lines showing a $\beta$-amylase diagram different from that of "Moisson"s.

\begin{tabular}{|c|c|c|c|c|c|}
\hline Origine 1975 & $2 n$ & $\mathrm{~N}^{0}$ & $\begin{array}{l}\text { Récoltes } \\
\text { analysées }\end{array}$ & $\begin{array}{c}\text { Type } \\
\beta \text {-amylasique }\end{array}$ & $\begin{array}{l}\text { Groupe } \\
\text { cytogénétique }\end{array}$ \\
\hline 129.9 .14 & 44 & m 1 & $1978-79$ & VIII & 5 \\
\hline 129.9 .17 & 43 & $\mathrm{~m} 3$ & 1977 & VIII & 5 \\
\hline 130.4 .14 & $42+t$ & m 12 & $1977-78-79$ & VIII & 5 \\
\hline 132.2 .6 & 44 & $\mathrm{~m} 52$ & $1977-78-79$ & II + VII, VIII $\left(^{*}\right)$ & 5 \\
\hline $143-15-16$ & 44 & $\int \mathrm{m} 102-11$ & $1977-78-79$ & II et VIII $(*)$ & $5 ?$ \\
\hline (double & & $\{\mathrm{m} \mathrm{102-22}$ & 1977 & II et VIII (*) & $5 ?$ \\
\hline monosomique) & & & 1978 & II et VIII (*) & 5 \\
\hline $145-9-3$ & 44 & m 128 & $1977-79$ & VIII & 9 ? \\
\hline $153-8-4$ & 43 & m 171 & $1977-78$ & $\mathrm{II}+\mathrm{VIII}, \mathrm{II}\left(^{*}\right)$ & 7 \\
\hline $176-13-3$ & 43 & m 294 & $1978-79$ & VIII & 5 \\
\hline $184-12-12$ & 43 & m 370 & $1978-79$ & $\mathrm{X}$ & $?$ \\
\hline
\end{tabular}

$\left(^{*}\right)$ Différents types obtenus selon les plantes ou les récoltes analysées. 
TABLEAU 2

Caractéristiques des lignées v ayant un diagramme B-amylasique différent de celui de "Moisson".

Characteristics of $v$ addition lines showing a $\beta$-amylase diagram different from that of "Moisson"s.

\begin{tabular}{|c|c|c|c|c|c|}
\hline Origine & $2 n$ & $\mathbf{N}^{\circ}$ & $\begin{array}{c}\text { Récolte(s) } \\
\text { analysée(s) }\end{array}$ & $\begin{array}{c}\text { Type } \\
\beta \text {-amylase }\end{array}$ & $\begin{array}{c}\text { Groupe } \\
\text { chromosomique }\end{array}$ \\
\hline $790-13-8$ & 44 & $\left\{\begin{array}{l}\vee 27 \\
\vee 30\end{array}\right.$ & $\begin{array}{l}78 \\
77\end{array}$ & $\begin{array}{l}\text { II } \\
\text { IX }\end{array}$ & $\begin{array}{l}4 \\
4\end{array}$ \\
\hline $792-13-10$ & 44 & v 86 & $77-79$ & $\mathrm{X}$ & 4 \\
\hline $792-2-1$ & 44 & v 104 & $77-79$ & IX & 4 \\
\hline 794-9-15 & 43 & v 130 & 78-79-80 & IX & $4 ?$ \\
\hline $795-10-23$ & 44 & v 132 & $77-79$ & IX & $4 ?$ \\
\hline \multirow{2}{*}{$795-5-3$} & & $\{\vee 136$ & $75-77-78-79$ & IX & 6 \\
\hline & 44 & v 137 & $75-77-79$ & IX & 6 \\
\hline $797-9-1$ & 44 & v 157 & $77-79-80$ & IX & 4 \\
\hline \multirow{2}{*}{$801-10-22$} & 43 & $\{$ v 176 & 75 & IX & 9 \\
\hline & 43 & I v 177 & 77 & II & 9 \\
\hline \multirow{3}{*}{$802-10-8$} & 43 & $\{\vee 183$ & $75-77-79$ & $\mathrm{X}$ et II & 7 \\
\hline & 43 & $\{$ v 186 & 78 & II & $2 n=42$ \\
\hline & & v 244 & 75 & IX & 5 \\
\hline \multirow[t]{2}{*}{$805-9-22$} & 44 & $\{$ v 246 & $75-77-79$ & $X$ et IX & 5 \\
\hline & & v 263 & $78-79$ & $\mathrm{X}$ & 5 \\
\hline \multirow{2}{*}{$805-5-18$} & & $\int \vee 260$ & $77-79$ & II & 5 \\
\hline & 43 & < 261 & $77-79$ & IX & 5 \\
\hline $805-8-21$ & 44 & v 269 & $78-79$ & IX & 4 \\
\hline $805-7-4$ & $?$ & v 271 & 78 & $\mathrm{X}$ & $2 n=42$ \\
\hline $810-10-13$ & 44 & v 353 & $78-79$ & IX & 4 \\
\hline $810-20-14$ & 44 & v 372 & $77-79$ & $\mathrm{X}$ & 4 \\
\hline $811-14-19$ & 43 & v 394 & $77-79$ & IX & $4 ?$ \\
\hline $811-3-11$ & 43 & v 396 & $77-78-79$ & IX & $4 ?$ \\
\hline
\end{tabular}

diagrammes correspondent à un marquage d'un chromosome additionnel $\mathbf{M}^{\mathrm{v}}$ : d'une part, les bandes $\mathrm{C}_{0}$ spécifiques de l'Aegilops ne sont pas observées chez ses progéniteurs et, d'autre part, les lignées appartenant à un même groupe défini d'après les critères cytologiques, morphologiques ou physiologiques n'ont jamais en totalité un diagramme identique.

En principe, une plante à $2 \mathrm{n}=44$ chromosomes donne une descendance à $2 \mathrm{n}=44$. Mais, comme 1 ou 2 plantes ont été analysées pour chaque récolte, on peut supposer que le diagramme II de type "Moisson" trouvé au sein de quelques descendances (m 52 et m 102) provient de grains à 42 chromosomes. Par ailleurs, il ne semble pas y avoir d'effet de dosage chromosomique : les plantes à 43,44 ou même 45 chromosomes des lignées v 137, v 261 et v 394 présentent des zymogrammes identiques (fig. 2). Il esi donc peu probable que des nombres chromosomiques différents expliquent toutes les hétérogénéités, notamment au niveau des différentes lignées d'un même groupe.

La comparaison des diagrammes obtenus avec les lignées $v$ et les lignées $m$ montre que la bande $C_{01}$ apparait parfois chez les lignées $v$ alors qu'elle n'est jamais obtenue chez les lignées $m$. D'une manière générale, la variabilité observée à partir des diagrammes des lignées $v$ est plus importante que celle des lignées $\mathrm{m}$. Ceci est peut être lié à l'utilisation d'une espèce «pont $» T$, aethiopicum, et à un nombre de rétrocroisements par «Moisson" plus faible chez les lignées v que chez les lignées $\mathrm{m}$.

Par ailleurs, chèz toutes les lignées $m$ et $v$ présentant un zymogramme VIII ou IX, il est intéressant de noter qu'on n'observe pas de constituants $\mathrm{C}_{3}$ (sous la dépendance du chromosome 4DL chez $T$. aestivum). On peut donc penser qu'il y a eu une recombinaison entre le chromosome 4D du blé tendre et le chromosome $4 \mathrm{D}^{v}$ de l'Aegilops. Dans quelques cas seulement (lignées de type $\mathrm{X}$ ), cette recombinaison ne se serait pas effectuée.
Il est donc vraisemblable que l'homéologie des chromosomes du groupe 4 , entre ces 2 espèces, soit assez grande.

\section{B. Peroxydases}

\section{Les blés et les Aegilops}

D'après leur composition peroxydasique les blés tendres ( $T$. aestivum) ont pu être divisés en 2 groupes (KOBREHEL \& GAUTIER, 1974). Les 2 isozymes majeures a-7 $D_{S}$ et d$7 \mathrm{~A}_{\mathrm{s}}$ (a, synthétisée par le bras court du chromosome $7 \mathrm{D}$, et $\mathrm{d}$ par le bras court du chromosome $7 \mathrm{~A}$ ) sont communes pour les 2 groupes; l'isozyme b est présente uniquement dans le groupe 1 et l'isozyme $c$ dans le groupe 2, la synthèse de cette dernière est assurée par le bras long du chromosome 4 B (c-4 B ) (KoBREHEL \& FEILLET, 1975).

Les blés tétraploïdes ne possèdent jamais l'isozyme a qui est synthétisée par le bras court du chromosome $7 \mathrm{D}$; par contre, ils ont tous les isozymes $\mathrm{c}$ et $\mathrm{d}$. Mais, tandis que l'intensité de ces isozymes est pratiquement constante chez les blés tendres, on peut observer chez les tétraploïdes une variation d'intensité relativement importante selon les espèces ou variétés analysées. Chez $T$. aethiopicum, les 2 peroxydases $c-4 B_{L}$ et $d-7 A_{S}$ sont intenses ; cette espèce a également 2 autres isozymes moins basiques et de faible intensité.

Ae. squarrosa (génome D) présente toujours la bande a$7 \mathrm{D}_{\mathrm{s}}$. Selon les souches, d'autres isozymes moins basiques et d'intensité variable peuvent être révélées, mais aucune d'elles ne semble correspondre à la bande principale des blés tendres d-7 $A_{s}$. Les 2 souches tétraploïdes étudiées ont des électrophorégrammes peroxydasiques identiques $(2 n=28$, DD). Les différences observées entre les souches diploïdes sont plus grandes qu'entre les espèces diploïdes et tétraploïdes. Le nombre et l'intensité des bandes peroxydasiques n'ont pas de rapport non plus avec le degré de ploïdie. 

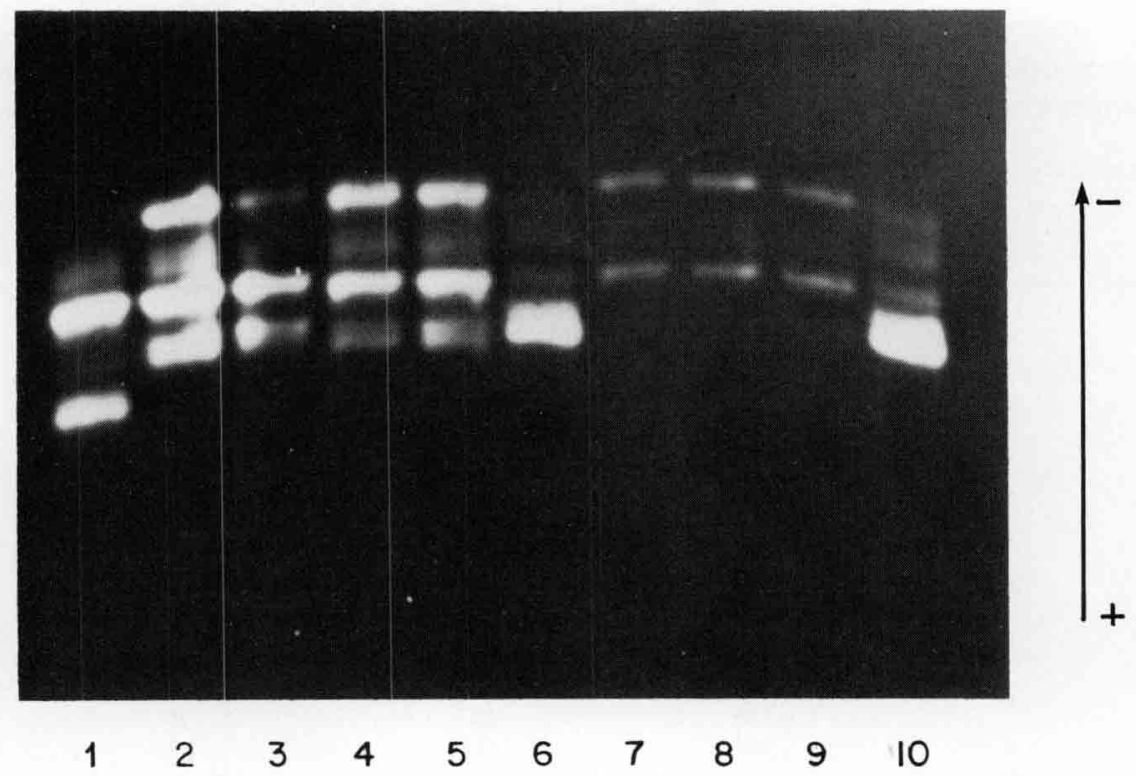

Figure 2

Zymogrammes $\beta$-anylasiques de lignées d'addition $v$ ayant un nombre différent de chromosomes.

$\beta$-amylase zymograms from $v$ addition lines with different chromosomal number.

1 - Témoin "Moisson

2- Lignée v 137.14.1.2. (44 chromosomes)

D'après les échantillons analysés, Ae. uniaristata $\left(\mathrm{M}^{\mathrm{u}}\right)$ ct Ae. comosa (M) auraient des compositions peroxydasiques plus homogènes, caractéristiques de l'espèce. Des 2 bandes principales d'Ae. uniaristata, l'une a la mobilité comparable à celle de $\mathrm{c}-4 \mathrm{~B}_{\mathrm{L}}$ et l'autre à celle de $\mathrm{d}-7 \mathrm{~A}_{\mathrm{S}}$. La $3^{\mathrm{c}}$ bande est moins mobile et d'intensité très faible (fig. 3). Ae. comosa a 2 bandes; la principale a une mobilité scmblable à d-7 $\mathrm{A}_{\mathrm{S}}$, l'autre est moins basique et de faible intensité, comparable à la $3^{\mathrm{e}}$ bande d'Ae. uniaristata (fig. 3).

Chez Ae. ventricosa, les diagrammes peroxydasiques sont composés de plusieurs bandes. L'isozyme a-7 $D_{S}$ y est toujours présente. La bande la plus intense se situe entre les
3 - Lignée v 137.14.1.6. (43 chromosomes) 4 - Lignée v 261.3.2.4. (43 chromosomes) 5 - Lignée v 261.3.2.9. (44 chromosomes) 6 - Témoin Ac. ventricosa 11

7 - Lignée v 394.2.29.3. (43 chromosomes) 8 - Lignée v 394.2.29.8. (44 chromosomes) 9 - Lignée v 394.2.29.2. (44 chromosomes) 10 - Témoin Ac. ventricosa 11

bandes $b$ et $c-4 B_{L}$ du blé tendre. Deux autres isozymes moins basiques d'intensité assez importante y sont présentes. La composition peroxydasique d'Ae. ventricosa semble être caractéristique de l'espèce; dans une seule souche ( $\left.\mathrm{n}^{\circ} 10\right)$, on a pu noter l'absence de la bande la plus basique (fig. 3).

L'amphiploïde $\mathrm{A}_{2}$ (Ae. ventricosa $\mathrm{n}^{\circ} 11 \times T$. aethiopicum $\left.\mathrm{n}^{\circ} 1 \mathrm{~A}\right)$ a plusieurs bandes peroxydasiques de forte intensité. Toutes les isozymes des parents Aegilops et blé semblent y être exprimées (fig. 4). La bande correspondante à $\mathrm{c}-4 \mathrm{~B}_{\mathrm{L}} \mathrm{y}$ est particulièrement intense.

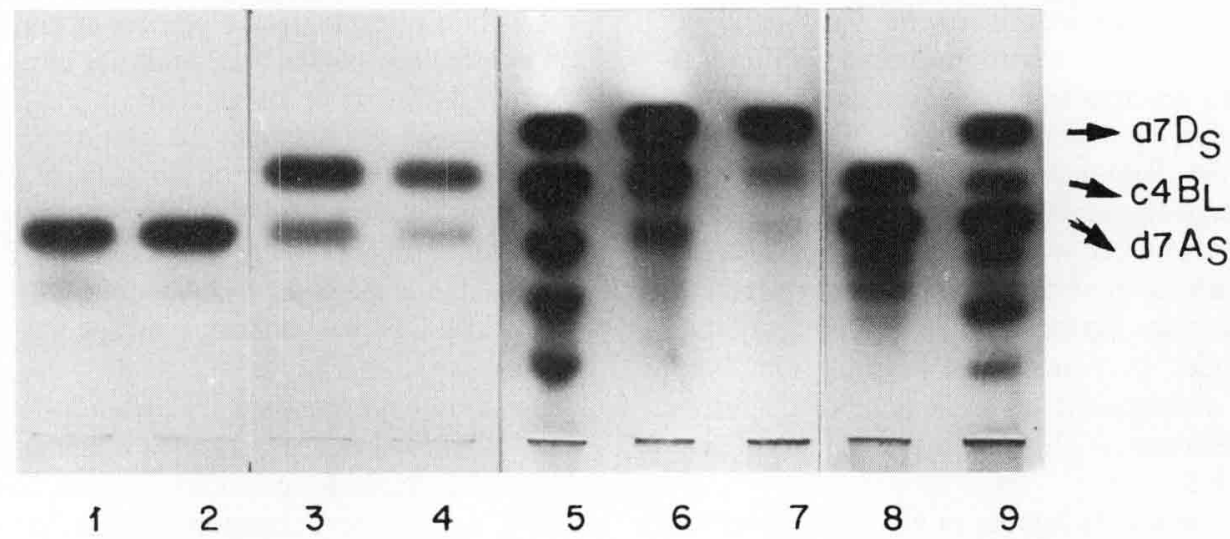

Figure 3

Zymogrammes peroxydasiques de différentes espèces de blé et d'Aegilops.

Peroxidase zymograms from different Acgilops and wheat species.

1 - Aegilops comosa $n^{\prime \prime} I$

2 - Aegilops comosa $n^{\prime \prime} 3$
3 - Acgilops uniaristata $n^{\prime \prime} I$

4 - Aegilops uniaristata $n^{\prime \prime} 2$

5 - Aegilops ventricosa $n^{\prime \prime} 7$

6 - Acgilops squarrosa $n^{\prime \prime} 9$

7 - Aegilops squarrosa $n^{\prime \prime} 20$

8 - Triticum acthiopicum $n^{\prime \prime} 1 A$

9 - Triticum aestivum (var. Moisson) 


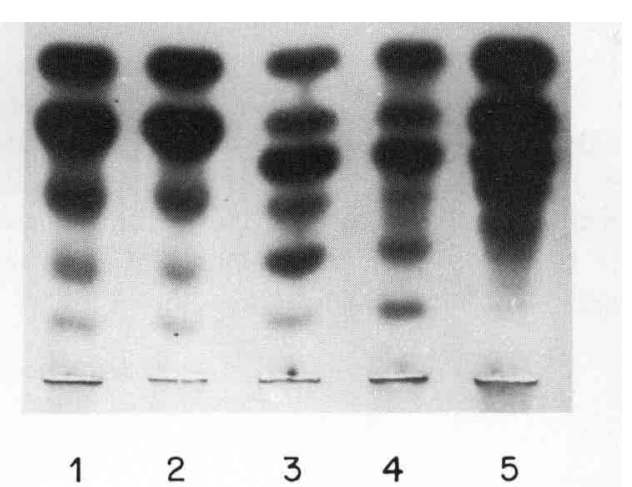

Figure 4

Principaux types peroxydasiques mis en évidence sur le matériel analysé.

Main types of peroxidase zymograms found in the analysed material.

1 - Ac. ventricosa $n^{\prime \prime} 7$

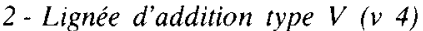

3 - Blé tendre var. Moisson

4- Lignée d'addition type $M$ (m 153)

5 - Amphiploïde $A$.

\section{Les lignées d'addition}

Les électrophorégrammes des lignées d'addition observés depuis 1976 se regroupent en 3 types principaux (fig. 4):

- le type $\mathrm{M}$ est pratiquement celui de la variété de blé tendre «Moisson». On n'a pas tenu compte de certaines différences présentes parfois au niveau des bandes les moins basiques et au niveau de la bande $c-4 \mathrm{~B}_{\mathrm{L}}$ dont l'intensité peut varier considérablement ;

- le type $\mathrm{V}$ qui a un diagramme similaire à celui d'Ae. ventricosa $\mathrm{n}^{\circ} 11$;

- le type A qui correspond au diagramme de l'amphiploïde $\mathrm{A}_{2}$.

La majeure partie des lignées $\mathrm{v}$ et $\mathrm{m}$ présente le diagramme de type M. L'absence d'une ou plusieurs bandes les moins basiques, ou des différences dans leur intensité au sein d'une même lignée, traduit une certaine hétérogénéité. On peut se demander si cela n'est pas dû, au moins partiellement, à l'état des germes plus ou moins développés du grain. En effet, certaines de ces isozymes peuvent provenir du germe (BENITO \& PEREZ dE LA VegA, 1979 ; BENITO et al., 1980).

Parmi les lignées $\mathrm{v}$, seules les lignées $\mathrm{v}_{4}$ et $\mathrm{v}_{9}$ expriment un zymogramme $\mathrm{V}$, quelle que soit l'année de récolte où les plantes sont analysées (tabl. 3). Ces lignées présentent un télosome à l'état mono- ou disomique. L'analyse comparée de leur niveau chromosomique et de leurs diagrammes montrent que le télosome additionnel n'est pas impliqué dans le marquage biochimique.

Le type $V$ se retrouve chez 2 lignées $m$. La lignée $m 26$ appartient au groupe 4 mais elle n'est pas fixée et présente un fort pourcentage de plantes à $2 \mathrm{n}=42$. La lignée $\mathrm{m} 408$ $(2 \mathrm{n}=42)$, bien qu'ayant perdu son chromosome additionnel, se rapproche du groupe 4 par son phénotype.

Il est peu probable que le diagramme $\mathrm{V}$ corresponde au marquage du chromosome additionnel car, d'une part, toutes les lignées du groupe 4 ne sont pas marquées et, d'autre part, les lignées $\mathrm{m} 26$ et $\mathrm{m} 408$, en dépit de la présence de plantes à $2 \mathrm{n}=42$, n'expriment pas de diagramme de type $\mathrm{M}$. Ce diagramme $\mathrm{V}$ traduit vraisemblablement la stabilisation de structures chromosomiques de recombinaisons entre les chromosomes $\mathrm{D}$ et $\mathrm{D}^{\mathrm{v}}$ du blé et de de l'Aegilops. Il est intéressant de souligner l'absence complète de la bande $\mathrm{d}-7 \mathrm{~A}_{\mathrm{S}}$ dans les lignées de type $\mathrm{V}$, alors qu'elle est la bande la plus importante par son intensité chez les blés tendres. On peut émettre plusieurs hypothèses: il peut s'agir d'une modification de la régulation de l'expression du chromosome 7 A qui se traduit par l'inhibition du gène contrôlant la synthèse de cette enzyme; la synthèse des peroxydases du blé est probablement sous contrôle monogénique (BENITO et al., 1980). L'absence de l'isozyme d-7 $\mathrm{A}_{\mathrm{S}}$ peut être aussi le résultat de recombinaison entre le génome $\mathrm{A}$ du blé et le génome $\mathrm{M}$ d'Ae. ventricosa. En effet, on peut supposer que cette recombinaison a comme conséquence l'inhibition au moins partielle de l'expression du chromosome $7 \mathrm{~A}$. La présence de la bande peroxydasique caractéristique des $A e$. ventricosa, celle qui est un peu plus basique que l'isozyme c-4 $\mathrm{B}_{\mathrm{L}}$, indique de toute évidence l'existence d'un fragment de chromosome d'Ae. ventricosa chez les lignées de type $\mathrm{V}$.

Le zymogramme de type $\mathrm{A}$ a été trouvé uniquement chez 4 lignées $\mathrm{m}$ dont 3 font partie du groupe 4 (tabl. 3). Il est tout aussi improbable que cela corresponde au marquage d'un chromosome additionnel en raison de l'hétérogénéité de réponse au sein d'une lignée et à l'intérieur d'un groupe.

TABLEAU 3

Caractéristiques des lignées $v$ et $m$ ayant un diagramme peroxydasique différent de celui de "Moisson ". Characteristics of $v$ and $m$ addition lines showing a peroxidase diagram different from that of "Moisson".

\begin{tabular}{|c|c|c|c|c|c|}
\hline $\begin{array}{c}\text { Origine } 74(\mathrm{v}) \\
\text { ou } 75(\mathrm{~m})\end{array}$ & $2 n$ & $\mathrm{~N}$ & $\begin{array}{l}\text { Récoltes } \\
\text { analysées }\end{array}$ & $\begin{array}{c}\text { Type } \\
\text { peroxydasique }\end{array}$ & $\begin{array}{c}\text { Groupe } \\
\text { chromosomique }\end{array}$ \\
\hline $789-12-10$ & $42+t$ & $\left\{\begin{array}{l}\times 4 \\
\vee 9\end{array}\right.$ & $\begin{array}{c}77-78-80 \\
77-80\end{array}$ & $\begin{array}{l}\text { V } \\
\text { V }\end{array}$ & $?$ \\
\hline $132-1-11$ & 44 & m 19 & 77 & $\mathrm{M}$ et $\mathrm{A}$ & 4 \\
\hline $132-8-15$ & & $\mathrm{~m} 26$ & 78 & V & 4 \\
\hline $143-12-5$ & 43 & $\mathrm{~m} 97$ & 78 & $M$ & 4 \\
\hline $143-12-12$ & $\begin{array}{c}42 \\
\text { (double } \\
\text { mono) }\end{array}$ & m 98 & 78 & A & 4 \\
\hline $183-2-15$ & 43 & m 348 & 77 & A & 4 \\
\hline $183-2-17$ & 43 & m 349 & 78 & $M$ & 7 \\
\hline $184-10-9$ & 44 & m 365 & $77-78$ & A. M & $?$ \\
\hline
\end{tabular}




\section{CONCLUSION}

Un double intérêt se dégage de ce travail : la grande variabilité détectée au sein des Aegilops à partir de quelques souches par espèce traduit la très grande diversité génétique de ce matériel. Par ailleurs, l'hétérozygotie structurale mise en évidence au niveau des lignées d'addition montre qu'elle se maintient très bien lorsqu'aucune pression de sélection ne s'exerce sur le caractère. Il est vraisemblable que ces lignées d'addition ne sont pas fixées pour la composition des enzymes étudiées. Par contre, elles semblent l'être pour les caractères sélectionnés et liés au chromosome additionnel (caractéristiques cytologiques, morphologiques ou agronomiques).

$\mathrm{Si}$, pour les gliadines (DosBa \& AUTRAN, 1983) et les $\beta$ amylases, l'hétérozygotie structurale se manifeste surtout au niveau des lignées $\mathrm{v}$ (chez lesquelles il y a eu seulement 2 rétrocroisements par "Moisson »), il n'en est pas de même pour les peroxydases. Leurs zymogrammes révèlent des types différents chez les lignées $m$ où 3 ou 4 rétrocroise- ments par «Moisson » ont été effectués et où l'espèce $T$. aethiopicum n'est pas intervenue. Il semble donc que la variabilité observée chez les lignées $v$ ne met pas en évidence l'hétérozygotie résiduelle liée à $T$. aethiopicum.

Par ailleurs, au niveau des $\beta$-amylases, nous confirmons les travaux de DeliBes et al. (1981) qui ont révélé des recombinaisons importantes entre les chromosomes $4 \mathrm{D}$ du blé et $4 \mathrm{D}^{\mathrm{v}}$ d'Ae. ventricosa, démontrant ainsi une forte homéologie des chromosomes du groupe 4.

Reçu le 23 juin 1982 Accepté le 18 février 1983.

\section{REMERCIEMENTS}

Nous remercions toutes les personnes qui ont contribué à ce travail et toutes celles qui ont aidé à sa publication.

Nous remercions particulièrement L. CHARBONNIER (I.N.R.A., Versailles) et J. SCHWENDIMAN qui ont apporté leurs critiques constructives.

\section{RÉFÉRENCES BIBLIOGRAPHIOUES}

Auriau P., Autran J. C., Charbonnier L., Doussinault G., Feillet P., Godon B., Grignac P., Joudrier P., Kobrehel K., Koller J., Rousset M., Rivallant S., 1976. Variabilité génétique de la composition des gliadines, gluténines, $\beta$-amylases, $\alpha$-estérases, peroxydases et phosphatases acides du blé (Triticum aestivum). Ann. Amélior. Plantes, 26 (1), 51-66.

Benito C., Perez de la Vega M., 1979. The chromosomal locations of peroxydase isozymes of wheat kernel. Theor. appl. Genet., 55, 73-76.

Benito C., Perez de la Vega M., Salinas J., 1980. The inheritance of wheat kernel peroxidases. J. Hered., 71, 416-418.

Bernard M., Autran J. C., Joudrier P., 1977. Possibilités d'identification de certains chromosomes du seigle à l'aide de marqueurs biochimiques. Ann. Amélior. Plantes, 27 (3), 355-362.

Brody T., Mendlinger S., 1980. Species relationships and genetic variation in the diploid wheats (Triticum, Aegilops) as revealed by starch gel electrophoresis. Pl. Syst. Evol., 136, 247-258.

Brown J. W. S., Kemble R. J., Law C. N., Flavell R. B., 1979. Control of endosperm proteins in Triticum aestivum (var. "Chinese spring ») and Aegilops umbellulata by homoelogous group 1 chromosomcs. Genetics, 93, 1, 189-200.

Caldwell K. A., Kasarda D. D., 1978. Assessment of genomic and species relationships in Triticum and Aegilops by Page and by differential staining of seed albumins and globulins. Theor. appl. Genet., 52, 6, 273-280.

Cauderon Y., Autran J. C., Joudrier P., Kobrehel K., 1978. Contrôle chromosomique des gliadines, des $\beta$-amylases et des peroxydases chez les lignées d'addition (Triticum aestivum $\times$ Agropyron intermedium). Ann. Amélior. Plantes, 28, 3, 257-267.

Delibes A., Otero C., Garcia-Olmedo F., Dosba F., 1981. Biochemical markers associated with two M' chromosomes from Aegilops ventricosa in wheat Aegilops ventricosa in wheat Aegilops addition lines. Theor. appl. Genet., 60, 5-10.

Dosba F., 1982. Les lignées d'addition blé - Ae, ventricosa. III Extraction et identification des lignées sur cytoplasme $A e$. ventricosa. Agronomie, 2 (5), 469-479.

Dosba F., Autran J. C., 1983. Les lignées d'addition blé Aev ventricosa. IV - Caractérisation des lignées et de leurs progénitcurs au moyen des électrophorégrammes des gliadines. Agronomie, 3 (6).

Dosba F., Doussinault G., 1978. Création de lignées de blé présen- tant les caractéristiques agronomiques favorables d'Aegilops ventricosa. Ann. Amélior. Plantes, 28, 4, 27-44.

Dosba F., Doussinault G., Rivoal R., 1978. Extraction, identification and utilization of the addition lines $T$. aestivum $\times$ Ae. ventricosa. Proc. 5th Int. Wheat Genet. Symp., New Delhi, 332-337.

Hart G E., Islam A. K., Shepherd K. W., 1980. Use of isozymes as chromosomes markers in the isolation and characterization of wheat barley chromosome addition lines. Genet. Res., 36, 3, 311325.

Irani B. N., Bhatia C. R., 1972. Chromosomal location of alcohol dehydrogenase gene(s) in ryc, using wheat-ryc addition lines. Genetica, 43, 2, 195-200.

Islam A. K., Shepherd K. W., Sparrow D. H. B., 1978. Production and characterization of wheat-barley addition lines. Indian Soc. Genet. Plant Breed., 365-371.

Joudrier P., 1980. Contrôle génétique de la $\beta$-amylase du grain de blé tendre. C.R. Acad. Sci., Paris, 291, D, 477-480.

Joudrier P., Bernard M., 1977. Responsabilité du génome D sur certaines isozymes $\beta$-amylase du grain de blé tendre. Ann. Amélior. Plantes, 27, 1, 35-47.

Joudrier P., Cauderon Y., 1976. Localisation chromosomique de gènes contrôlant la synthèse de certains constituants $\beta$-amylasiques du grain de blé tendre. C.R. Acad. Sci., Paris, 282, D, 115-118. Khakimova A. G., 1979. Immunochemical and electrophoretic analysis of ethanol soluble seed proteins of Aegilops L. species with D genomc. Tr. Prikl. Bot. Genet. Sel., 63, 3, 38-49.

Kobrehel K., 1978. Identification of chromosome segments controlling the synthesis of peroxidases in wheat seeds and in transfer lines with Agropyron elongatum. Can. J. Bot., 56, 10911094.

Kobrehel K., Feillet P., 1975. Identification of genomes and chromosomes involved in peroxidase synthesis of wheat seeds. Can. J. Bot., 53, 2336-2344.

Kobrehel K., Gautier M. F., 1974. Variability in peroxidase isozymes in wheat and related species. Can. J. Bot., 52, 755-759.

Konarev A. V., 1979. Electrophoretic and immunochemical study of the genome specific proteins in wheat and Agropyron. Byull. Vses. Ord. Lenina, 92, 5-10.

Nagayoshi T., Mochizuki A., 1971. Amylase isozymes in wheat lines with additional chromosomes for Agropyron elongatum. Kobe Daisaku Nogakubu Kenkyu Hokoku, 9, 1-2, 71-74. 